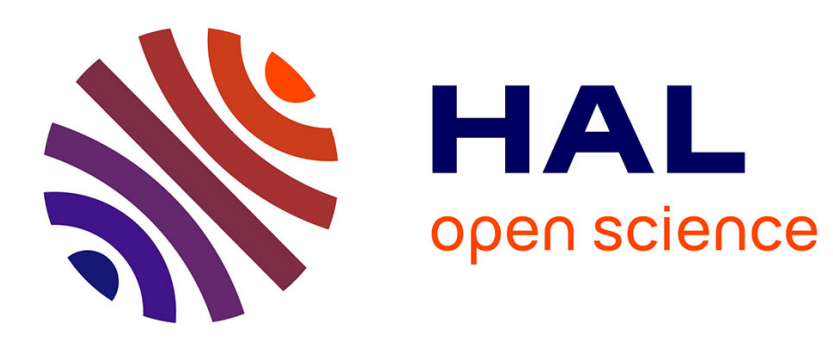

\title{
Pantographic 2D sheets: Discussion of some numerical investigations and potential applications
}

Francesco Dell'Isola, Alessandro Della Corte, Ivan Giorgio, Daria Scerrato

\section{To cite this version:}

Francesco Dell'Isola, Alessandro Della Corte, Ivan Giorgio, Daria Scerrato. Pantographic 2D sheets: Discussion of some numerical investigations and potential applications. International Journal of NonLinear Mechanics, 2016, 80, pp.200-208. hal-01287032

\section{HAL Id: hal-01287032 https://hal.science/hal-01287032}

Submitted on 11 Mar 2016

HAL is a multi-disciplinary open access archive for the deposit and dissemination of scientific research documents, whether they are published or not. The documents may come from teaching and research institutions in France or abroad, or from public or private research centers.
L'archive ouverte pluridisciplinaire HAL, est destinée au dépôt et à la diffusion de documents scientifiques de niveau recherche, publiés ou non, émanant des établissements d'enseignement et de recherche français ou étrangers, des laboratoires publics ou privés. 


\title{
Pantographic 2D sheets: Discussion of some numerical investigations and potential applications
}

\author{
Francesco dell'Isola ${ }^{a, c}$, Alessandro Della Corte $^{\mathrm{b}, *}$, Ivan Giorgio ${ }^{a, c}$, Daria Scerrato $^{c}$ \\ a Department of Structural and Geotechnical Engineering, Università di Roma La Sapienza, 18 Via Eudossiana, Roma, Italy \\ b Dactoral School in Theoretical and Applied Mechanics. Università di Roma La Sapienza, 18 Via Eudossiana, Roma, Italy \\ ${ }^{`}$ MeMocS, International Research Center for the Mathematics \& Mechanics of Complex Systems, Università dell'Aquila, Italy
}

\begin{abstract}
A B S T R A C T
The study of generalized continuum models through the numerical investigation of discrete systems considered as an approximation to a homogenized continuum limit is nowadays a well-known research approach in mechanics. In the present paper, a system constituted by a large number of beams interconnected via ideal hinges, called here a pantographic sheet, is considered, and some numerical simulations concerning the static and dynamic analysis of the system are presented and discussed. The observed behavior significantly differs from what one would expect from ordinary first gradient continuum models. Moreover, interesting application possibilities entailed by the specific characteristics of the structure, and in particular by the strong non-linear behavior of the mechanical variables, are discussed.
\end{abstract}

\section{Introduction}

The rapid development of increasingly powerful computational tools is having a deep influence on scientific research in non-linear mechanics. Indeed, the availability of highly performing software and processors, and also the findings in the theoretical study of several problems (e.g. minimization problems entailing the possibility of instabilities), allowed such a marked quantum leap in the scope of numerical investigations to affect even the status of some important and deep theoretical questions. A theoretical problem which can be addressed in a more efficient way by using the whole arsenal of today available computational tools is constituted by the long debated issue concerning higher-gradient theories in continuum mechanics.

Mechanical interactions are usually thought of to be caused by surface contact forces, which one can mathematically express via the stress tensor $\sigma$. This is of course true when dealing with first gradient continua, but not anymore, in general, when one considers models in which deformation energy depends on second and higher gradients of the displacement. While the debate about the meaningfulness of such generalized models was limited, until a few years ago, to rather abstract theoretical (and even philosophical) reasoning. it is today possible to numerically study some

\footnotetext{
- Corresponding author.

E-mail addresses: francesco.dellisolaeuniroma l.it (F. dell'Isola), alessandro,dellacorteలuniroma 1.it (A. Della Corte). ivan.giorgiopuniroma 1.it (1. Giorgio), daria.scerratoouniroma 1.it (D. Scerrato).
}

specific examples which can provide concrete evidences supporting the usefulness of higher gradient theories.

We will consider a 2D mechanical system constituted by a great number of ordinary Euler beams forming two orthogonal arrays, showing some interesting results which suggest that a homogenized limit for the considered system will present a richer behavior than that allowed by a first gradient model. Moreover, we want to underline some of the mechanical characteristics of the structure and to outline some of its potential applications. The system considered herein was first investigated in [1,2], where simulations about linearized behavior were shown. In the present paper, we considered simulations including geometric non-linearities, and a broader set of variables was studied.

The study of generalized continua through the numerical investigation of discrete systems considered as an approximation to a homogenized continuous limit is nowadays a well-known research approach in mechanics (see for instance [3-7] for applications to structures presenting similarities with the one considered herein), and the consideration of both a continuous and a discrete case in the same context is increasingly frequent, also due to the recent great appealing of nanotechnology (see e.g. the cases analyzed in $[8-11])$. The consideration of suitable discrete mechanical models can lead, in a rather straightforward manner. to the emergence of properties and behaviors which are characteristic of higher gradient models, thus allowing the reasonable conjecture that their homogenized limit is conveniently described by a higher gradient continuum model. of course, the existing literature on higher order continua is very wide - the reader can 
refer for example to [12-19]. It is also well known that considering additional kinematic descriptors, which leads to the so-called micromorphic continuum models, can be seen as a generalization of higher gradient theories. For instance, in the case of the system here considered, one could use an independent kinematical descriptor to account for the geodesic (in-plane) bending of the beams. The wide generality of micromorphic continua makes them one of the most powerful theoretical tools to study the behavior of very complex mechanical systems; on micromorphic continua a classic reference is [20], while a review of some more recent results is [21,22]. The state-of-the-art literature clearly shows that higher gradient models and micromorphic continua are nowadays very relevant and vital research areas, and therefore lines of investigations potentially capable to address some related foundation issues can be of great interest.

Concerning the numerical investigation of systems of the type herein considered, one should take into account that it can very easily lead to non-trivial problems (see for more details [23,24]), in particular when considering peculiar geometrical configurations; however, recent results in numerical analysis of similar cases involving nets of beams or related homogenized system can prove useful also in our context. Recent developments in finite element analysis have indeed provided sharp tools for addressing problems in which large displacements and deformations are involved (for applications of Isogeometric Analysis for discrete systems and the reader is referred e.g. to [25-28], while for application of freelocking finite element analysis to continuous systems see e.g. $[29,30])$. Another typical source of troubles in this context is the onset of instabilities of various kinds, and also in this direction some recent results (as those exposed e.g. in [31-35]) have strongly helped the possibility of novel theoretical developments. The development of new numerical tools can also have consequences on the degree of interest of some subjects. The classic theory of beams by Euler considered in the present paper, for instance, still offers space for far from trivial investigation, especially considering that today's computational tools allow the study of exotic geometrical or elastic features (as done for example in [36-39]). On the whole, the availability of new numerical results and methods seems to suggest that it is probably the right moment to try to systematically address via numerical investigation the aforementioned theoretical issues.

Let us start by briefly recalling the main features of the considered system (for a more detailed description see [1]). Each beam belonging to one of the two arrays is connected with hinges to the beams belonging to the other array, thus resulting in a structure which is natural to call a pantographic sheet. Notice that each beam is continuous throughout all its length. The structure is therefore actually constituted by arrays of beams, whereas the presence of hinges interrupting the continuity of the beams would have led to a typical truss. The effect of hinges is, then, only that of constraining two discrete sets of points, belonging respectively to the two families of the beams, to have the same displacement (see Eq. (2)). In Fig. 1 the reference configuration $C^{*}$ is represented. Beams are graphically represented by lines, belonging to two families $(\alpha, \beta)$ of parallel and equally distanced beams (with steplength $d$ ), mutually orthogonal in the reference configuration. $C^{*}$ has a rectangular shape with sides length $\ell=\sqrt{2} L d$ and $b=\sqrt{2} W d$, and in it the two families $\alpha$ and $\beta$ intersect at $45^{\circ}$. As
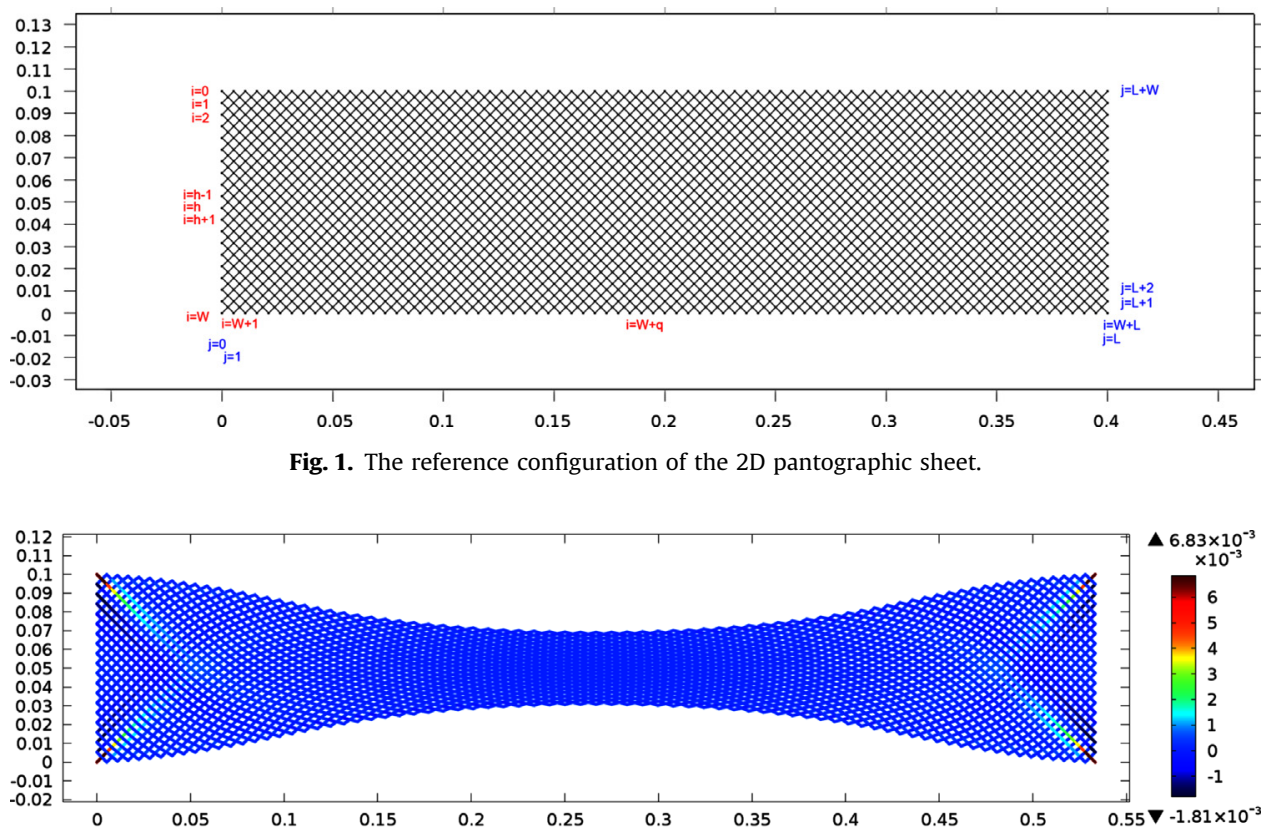

Fig. 2. Axial strain evaluated at the cross-section's beam centroid.

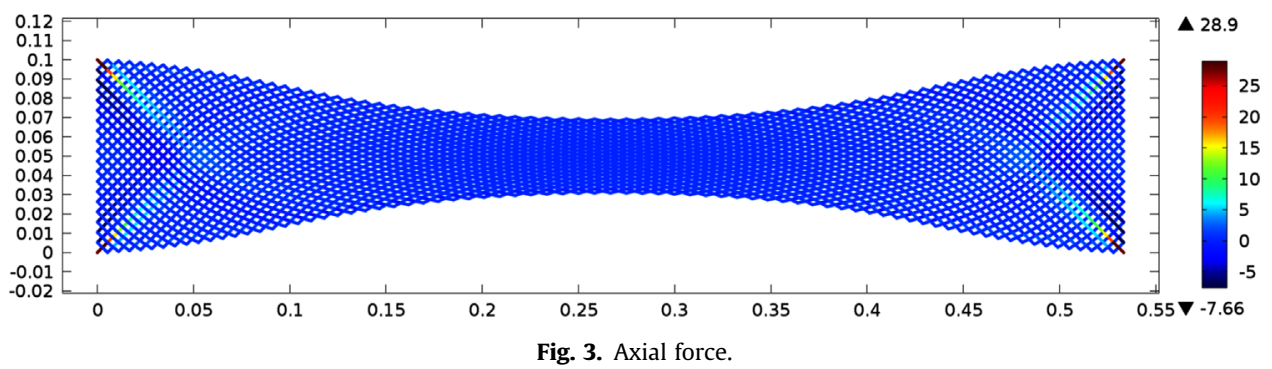

Fig. 3. Axial force. 

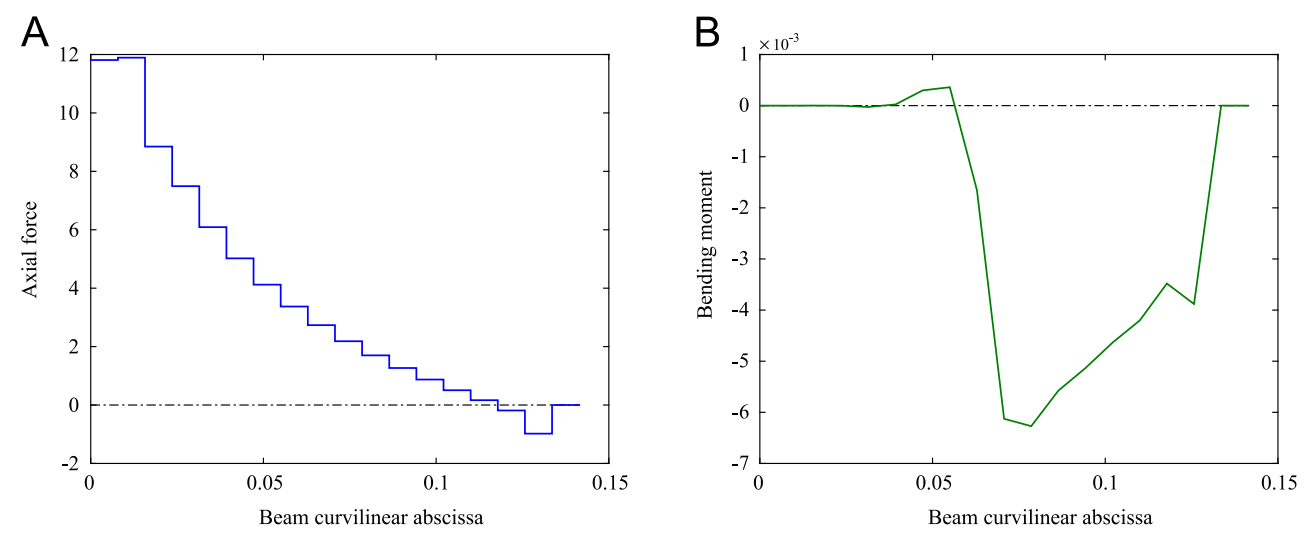

C

D
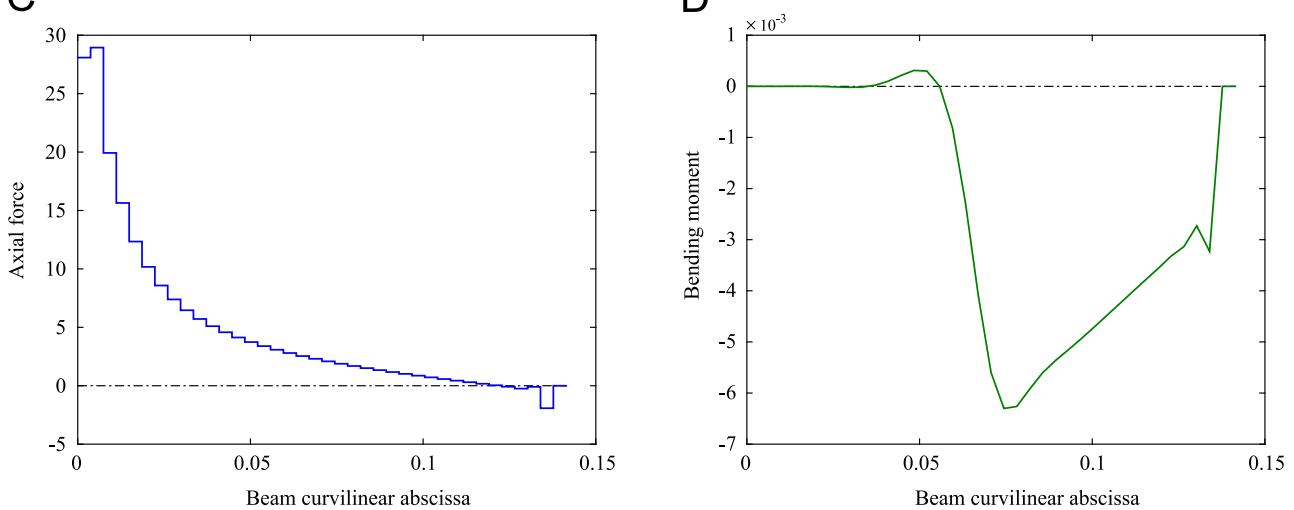

E

$\mathrm{F}$
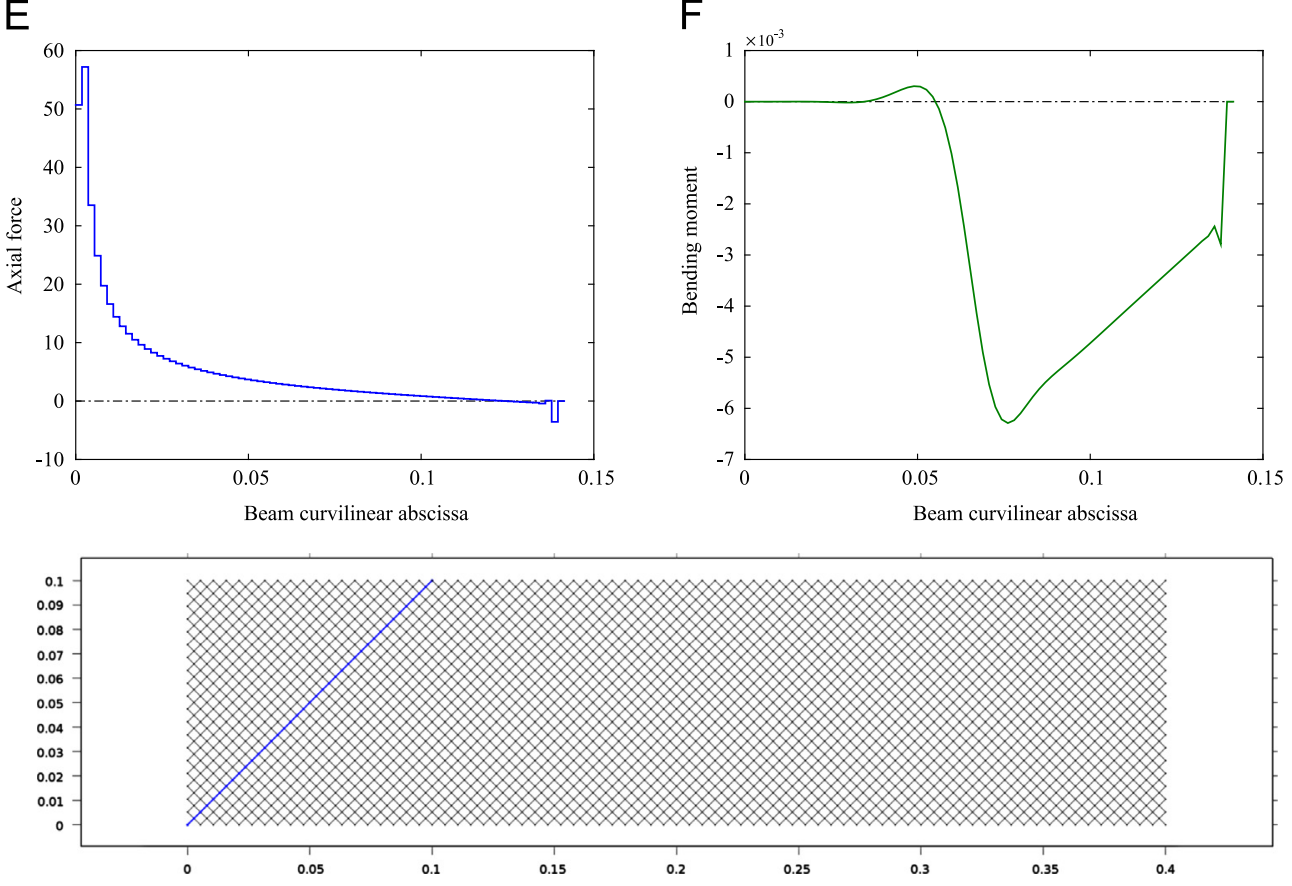

Fig. 4. Internal forces along the marked beam.

graphically indicated in the figure, beams belonging to $\alpha$ and $\beta$ are respectively denoted with the integer indexes $i$ and $j(0 \leq i \leq L+W$, $0 \leq j \leq L+W$ ). Every intersection is therefore uniquely determined by a pair of indexes $(i, j)$.

Each beam has a deformation energy density given by:

$\mathcal{E}=\frac{k_{M}\left(\phi^{\prime}\right)^{2}+k_{N}\left(w^{\prime}\right)^{2}}{2}$
Here $\phi$ and $w$ are respectively the magnitudes of the rotation of the cross section of the beam and of the axial displacement $\mathbf{w}$ with respect to reference configuration of the beam, and $k_{M}, k_{N}$ are respectively bending and axial stiffness coefficients.

Dots in Fig. 1 represent hinges that are kinematically defined by the following vector equation:

$\mathbf{u}_{b}(i, j)+\mathbf{w}_{b}(i, j)=\mathbf{u}_{c}(i, j)+\mathbf{w}_{c}(i, j)$, 
where $\mathbf{u}$ is the transverse displacement with respect to the reference configuration of the beam. The previous relation must hold $\forall b \in \alpha, c \in \beta$, and for all integers $i, j$ such that $0 \leq i \leq L+W$ and $0 \leq j \leq L+W$.

The actual configuration $C^{t}$ is characterized, with respect to $C^{*}$, by 'small' deformations and (possibly) 'large' displacements. These geometric and kinematic characteristics of the system lead (even when considering a linearized beam model) to the onset of sharp non-linearities, as we will see below.

\section{Static analysis}

In this section a static study of the considered structure is provided for a reference configuration $C^{*}$ with a shape ratio $\ell / b=4$. All numerical simulations were performed with COMSOL Multiphysics.

We prescribed an imposed horizontal displacement $\Delta u$ on the points belonging to the right side of $C^{*}$. Our value for $\Delta u$ is $1 / 3$ of the length $\ell$ of the basis of $C^{*}$. The left side is clamped. In Fig. 2 the axial deformation of the system in the given conditions is shown. The deformation field of the beam axes for $\alpha$ and $\beta$ is also plotted. Right and left sides are undeformed, and it can be seen that, as it is well known in the continuous case, two triangle-shaped regions close to the sides are stressed at a very low level, while a higher concentration of strain is observed in the vertexes and gradually decreases along the sides of the two aforementioned triangular regions. These results are confirmed by well-established experimental data (see e.g. $[40,41]$ ).

In Fig. 3, the axial force relative to the previous simulation is plotted. As one could expect from the previous case, a maximum

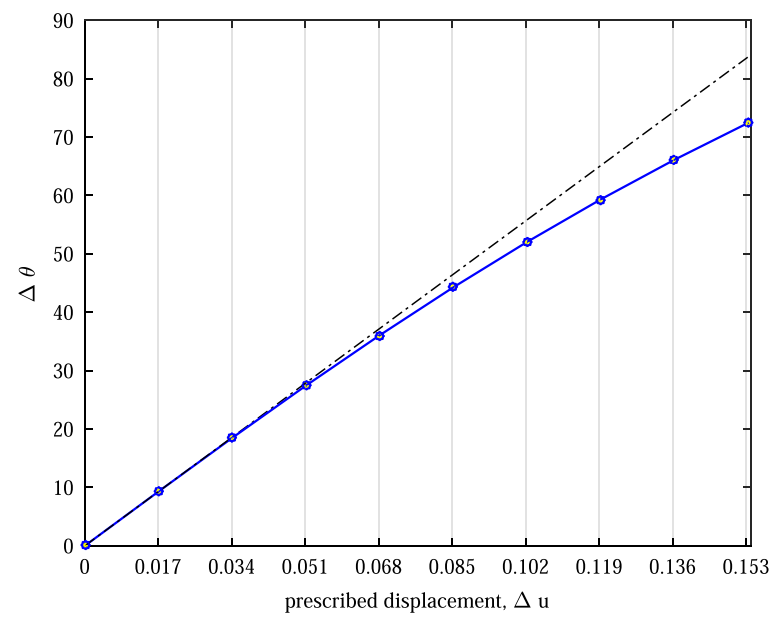

Fig. 5. Variation of angle between the two families of beams at the center of the pantographic sheet as a function of the prescribed displacement. A comparison with the linear regime (dot-dashed line) is provided. value for the magnitude of the axial force is reached at the vertexes, and moreover the peak is quite sharp.

In the next group of static simulations (see Fig. 4), we considered a particular beam $(j=0$, highlighted in the bottom panel) and studied different mechanical variables related to it. This specific beam was chosen because it is representative of the most stressed region, as observable from the previous figure. The three cases are relative to an increasingly dense beams configuration. Panels $\mathrm{A}-\mathrm{B}, \mathrm{C}-\mathrm{D}$, and $\mathrm{E}-\mathrm{F}$ are indeed referred to an undeformed configuration $C^{*}$ in which the step-length is respectively $2 d, d$ and $d / 2$ (the second case corresponds to the reference configuration shown in Fig. 1). In panels $A, C$ and $E$, the axial force $(\mathrm{N})$ is plotted versus beam curvilinear abscissa $(\mathrm{m})$. Also in these cases a prescribed displacement $\Delta u$ of magnitude $\ell / 3$ has been imposed to the points belonging to the right side, while the left side is clamped. As one can see, the force is piece-wise constant, which is clearly due to the fact that it is exerted by the hinges. As the number of beams increases, the plot seems to converge to a highly non-linear curve, having a sharp maximum near to 0 . This characteristic can have interesting implications of the applications, since it means that the considered kind of structure has a very well predictable tensile fracture region. The maximum observed value, moreover, is dependent on the number of considered beams (respectively $\approx 12 \mathrm{~N}, \approx 30 \mathrm{~N}$ and $\approx 60 \mathrm{~N}$ in panels $\mathrm{A}, \mathrm{C}$ and $\mathrm{E}$ ).

In panels $B, D, F$ the bending moment $(\mathrm{N} \mathrm{m})$ is plotted versus beam curvilinear abscissa $(\mathrm{m})$. In this cases, the trend is piece-wise linear, with a 0 value at the beginning, while a marked minimum is visible around the middle of the beam. As the number of beams increases, the plot seems to converge, also in this case, to a strongly non-linear curve. A typical edge effect is observable around the end of the beam. As there are no hinges in the end points of the beams, upper end points are unstressed.

In the next simulation, the angle $\theta$ that the beams form at the crossing points is considered. In particular, the difference $\Delta \theta$

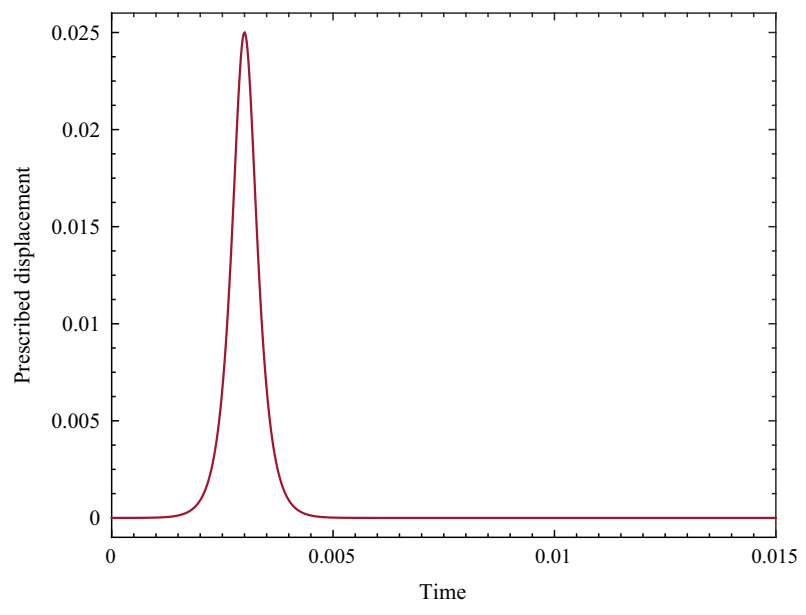

Fig. 7. Prescribed displacement.

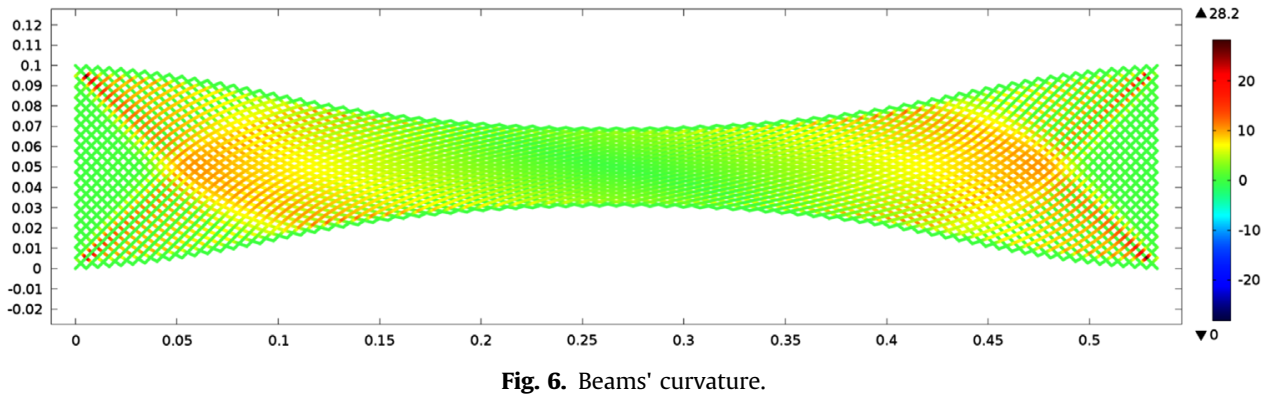



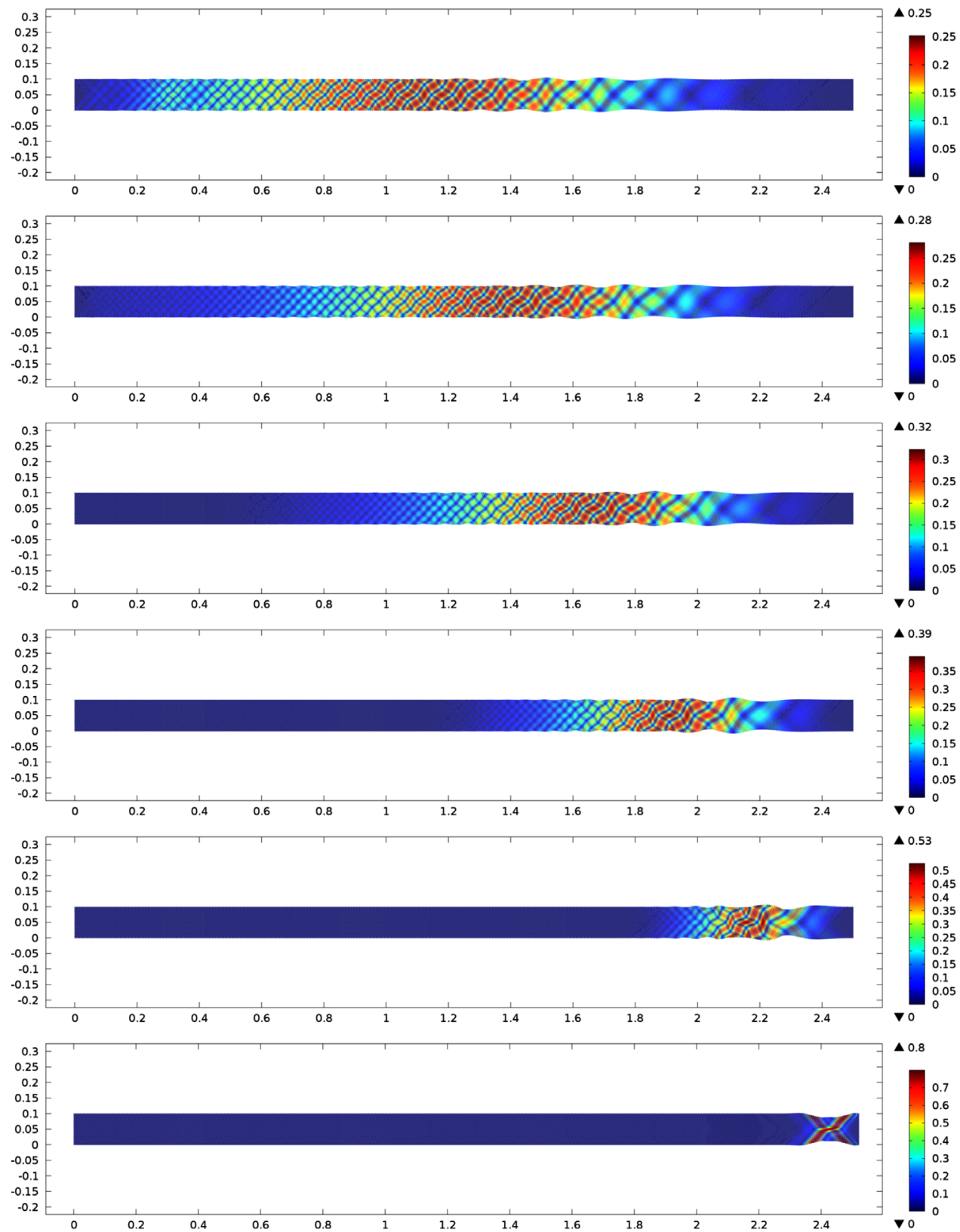

Fig. 8. Propagating wave as a consequence of an imposed time-dependent longitudinal displacement at the right side of the sheet.

(degrees) between the actual value of $\theta$ at the center point of the structure and the reference value $\theta_{0}=90^{\circ}$ at the same point is plotted versus the prescribed displacement $\Delta u(\mathrm{~m})$. The considered values for $\Delta u$ are 10 equally spaced values between 0 and $0.153 \mathrm{~m}$. In Fig. 5, a comparison with a linear behavior (dot-dash line) is shown. Also in this case a significant non-linearity is observable. The fact that the difference $\Delta \theta$ can have large values in this kind of loading is of course of important in case ideal hinges are replaced by hinges with an associated rotational stiffness, which is true in almost every real-world case.

In Fig. 6 the curvature $\left(\mathrm{m}^{-1}\right)$ of the beams is plotted, again with a prescribed displacement $\Delta u=\ell / 3$ imposed on the points belonging to the right side and a clamped left side. The behavior in this case is highly significant for our purposes, because it shows a richness which is not compatible with a classic first gradient model. Indeed, two practically undeformed regions are connected (smoothly along each beam) via a 'boundary layer' which is markedly deformed. It is therefore reasonable to conjecture that the homogenized limit of the considered structure is best modeled by a second gradient continuum.

\section{Analysis of some dynamic cases}

Discrete systems' dynamics can be very complex, possibly involving all the exotic and peculiar behaviors one can find studying continuum systems (for a description of some of the possible dynamic phenomena like unusual wave propagation or dissipation which may occur in this kind of structures, the reader can refer to e.g. [42-45]).

Four dynamic cases are considered in this section for a reference configuration $C^{*}$ with a shape ratio $\ell / b=25$. In Fig. 7 , the time history of an impulsive time-dependent displacement $u(t)$, having its maximum at $t=0.003 \mathrm{~s}$, is shown. The displacement $u(t)$ is then imposed to the points belonging to the right side of the structure, while the left side is clamped. The consequent evolution 

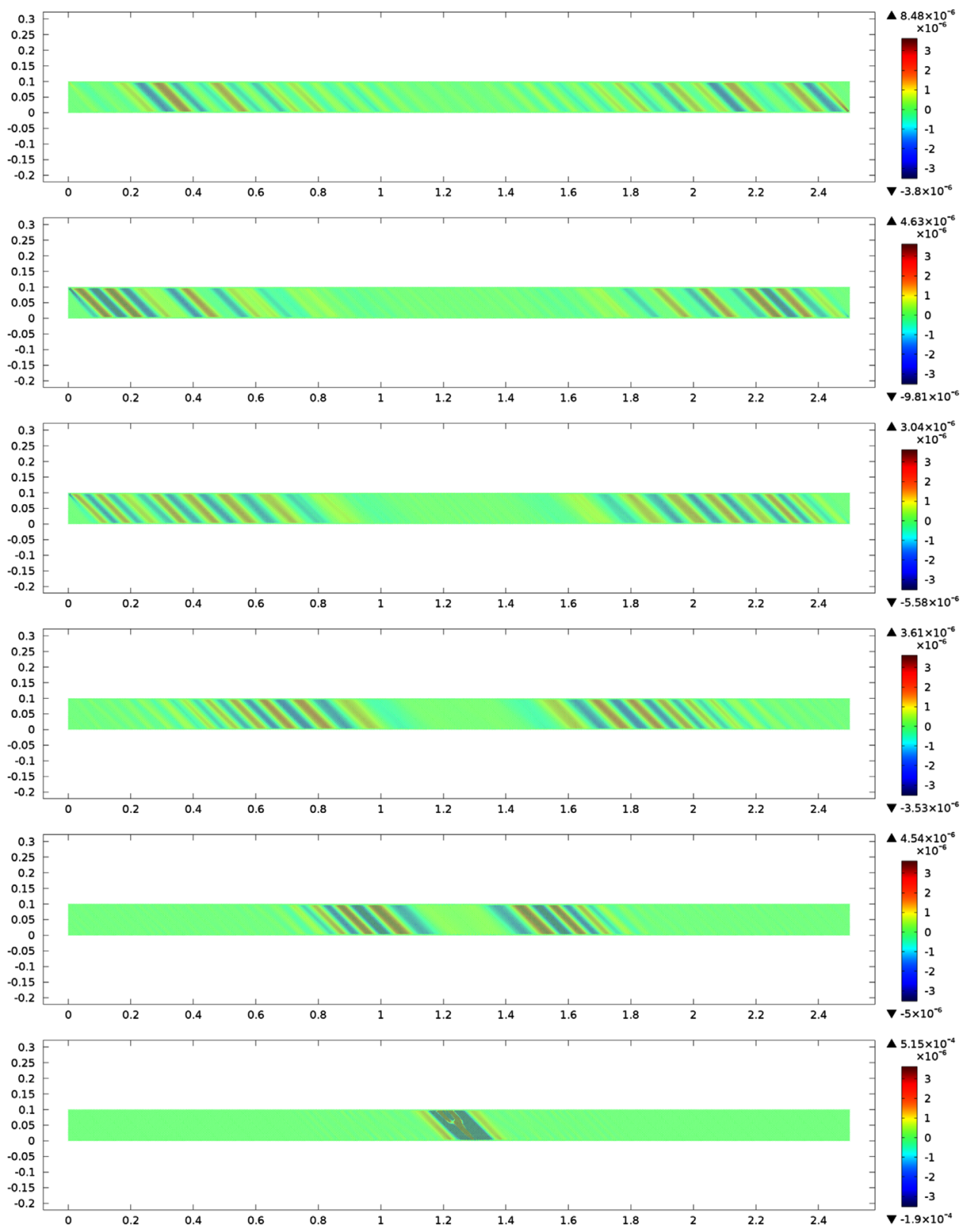

Fig. 9. Propagating wave as a consequence of an imposed time-dependent system of three self-equilibrated forces.

of the system is shown in Fig. 8, where the total rotation of the cross section of the beams is plotted. The six snapshots are relative to time steps of $0.00225 \mathrm{~s}$ starting from the time at which the displacement reaches its maximum value $\left(u_{\operatorname{Max}}=0.025 \mathrm{~m}\right)$. A quite standard wave propagation arises, strongly resembling that corresponding to a continuum case. Dispersion is observable, as one can judge from the increasing length of the perturbed area.

In the next case, we wanted to check how the structure responds to a loading which in its continuum limit resembles a (bending) couple. We indeed considered a system of three impulse-like forces $F_{1}, F_{2}, F_{3}$, which are time-dependent and selfequilibrated (having both resultant and resultant moment equal to zero) in every instant, and have maximum values of $1 \mathrm{~N}\left(F_{1}\right)$ and $1 /$ $2 \mathrm{~N}\left(F_{2}, F_{3}\right)$. They are applied to the end points of three contiguous beams in the center of the system, i.e. $F_{2}$ at point $(i=0, j=L / 2-1)$, $F_{3}$ at point $(i=0, j=L / 2+1)$ and $F_{1}$ at point $(i=W+L / 2, j=L / 2)$. The three forces are oriented along the direction of the beams belonging to one of the two families $(\alpha)$. The wave propagation originated in this way is particular interesting for our purposes, as the axial deformation is negligible in all the time history, while a significant bending perturbation is traveling (see Fig. 9). This also suggests that the homogenized limit of the considered system requires a second gradient model.

The third dynamic case that we considered concerns the simultaneous prescription of two opposite time-dependent displacements on the two sides of the system in the same direction of the $\alpha$-family beams. The trend of the time history of the prescribed displacement is the same as that displayed in Fig. 7. In Fig. 10, six snapshots are presented with a time step of $0.0027 \mathrm{~s}$, starting from the time corresponding to the maximum value of the prescribed perturbation $\left(u_{\text {Max }}=0.01 \mathrm{~m}\right)$. The rotations of the cross section of the beams are graphically represented. Also in this case considerable dispersion is observable, and the propagation and the interaction of waves closely resemble analogous cases in continuum media.

Finally, we considered in Fig. 11 the case of two pantographic sheets connected by an array of hinged longitudinal beams $k_{M}=1.96$ $\times 10^{-2} \mathrm{~N} \mathrm{~m}^{2}$ and $k_{N}=7.85 \times 10^{4} \mathrm{~N}$; specifically, these beams are incorporated in an elastic medium to prevent rigid motion (indeed, 

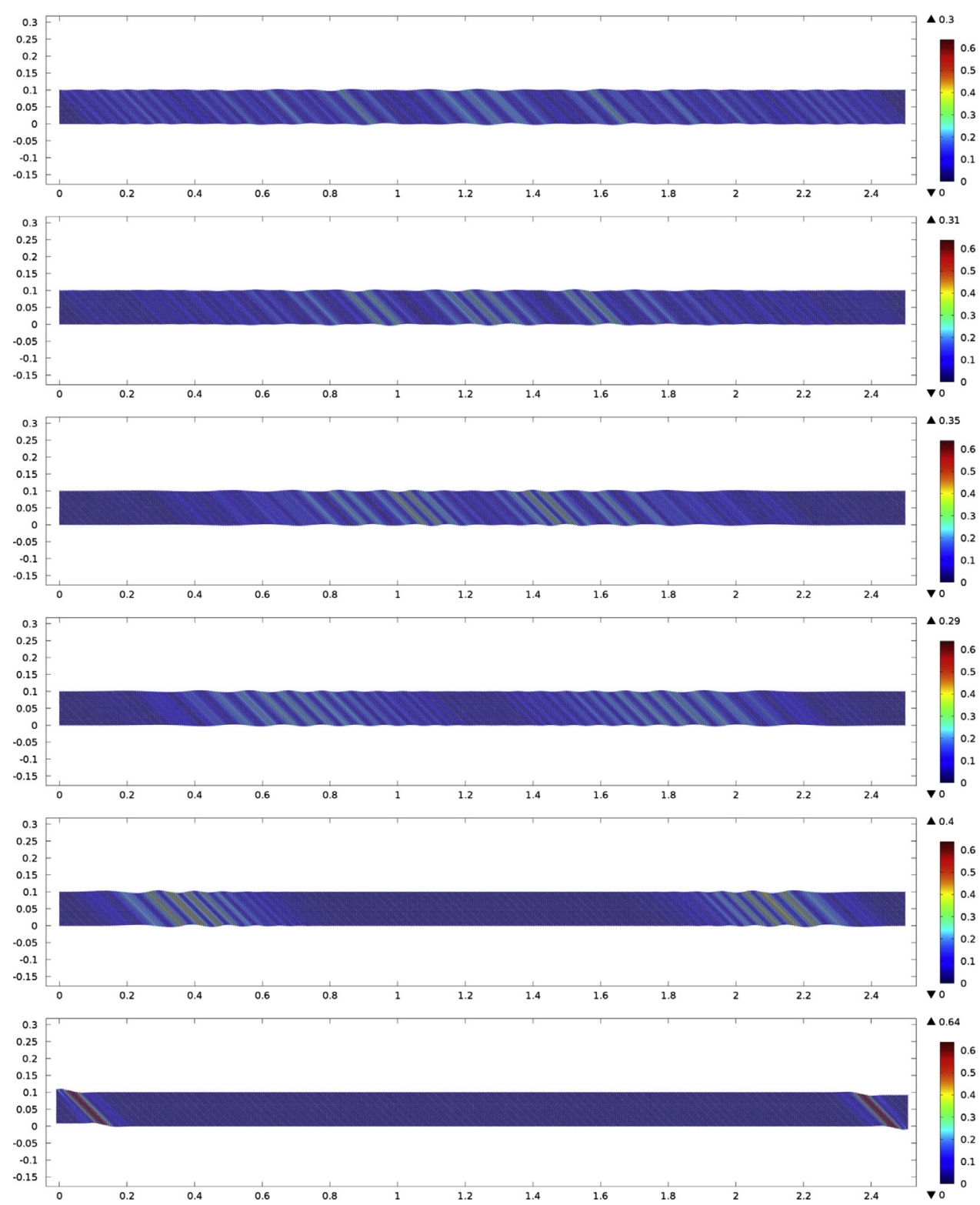

Fig. 10. Propagating waves as a consequence of two opposite prescribed time-dependent displacements at both sides of the sheet.

without this matrix, we would have an array of four-bar linkages). The displacement is prescribed to the points belonging to the right side and is oriented along $\alpha$, and has a maximum value of $0.02 \mathrm{~m}$; the time-step is the same as for the previous simulations. As one can see, there is practically no wave propagation beyond the discontinuity at the center, and this circumstance suggests a potentially useful employment of pantographic sheets with internal discontinuities in the designing of filters capable to effectively damp vibrations.

\section{Conclusions}

It is well known that the behavior exhibited by systems constituted by very simple interacting objects can be much more complex and rich than one could expect from the single constitutive element. In the present work, the investigated mechanical system was indeed constituted by ordinary (non-linearized) Euler beams displayed in a geometrically periodic configuration. The numerical analysis of the structure showed peculiar (strongly non-linear) trends for the axial force, the bending moment and the relative rotation between the beams.

The technological appealing of pantographic sheets is linked to the possibility of combining high mechanical strength with lightness and flexibility. The structure considered in the present paper has indeed a potentially very attractive strength-to-weight ratio (especially in case suitable materials, like kevlar or carbon fiber, are employed). Moreover, the pantographic sheet is in our opinion particularly interesting since it adds to this feature a very wellpredictable fracture behavior. Indeed, the static analysis above performed showed that not only the maximum values for the tensile stress and bending moment are quite concentrated in specific areas, but also that the peak shown there is very sharp, thus allowing a very simplified checking procedure for the mechanical resistance of real-world prototypes.

The need to investigate the theory of the homogenized limit of structures with the mechanical and geometrical characteristics here described comes from two directions. First of all, the realization of real pantographic sheets made of a very large number of beams, and 

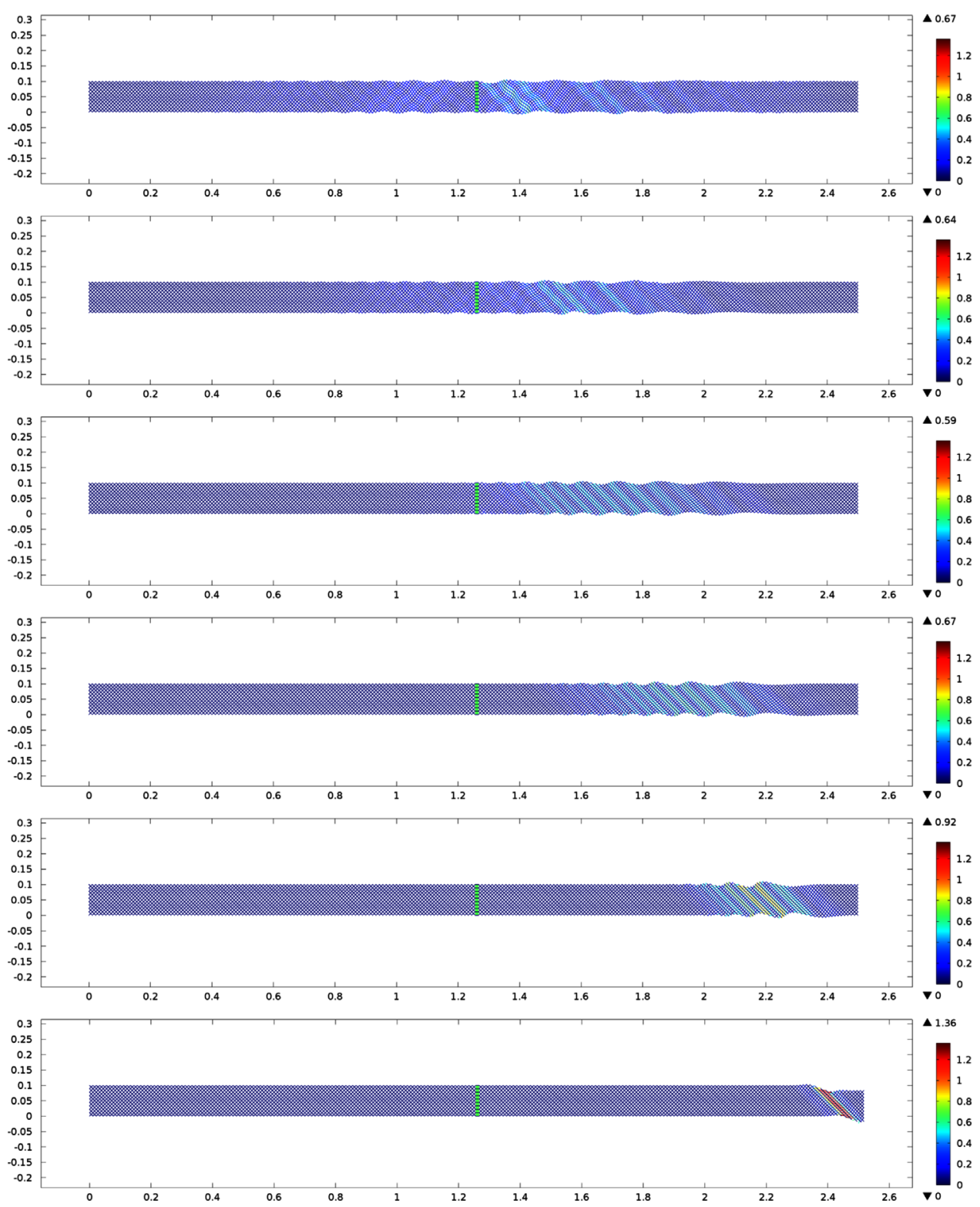

Fig. 11. Wave propagation in two sheets connected at the middle by an array of hinged longitudinal beams incorporated in an soft elastic medium.

in particular the possibility to consider even nano-objects and the consequent desire to numerically predict their behavior, would lead to a sharply increasing computational complexity, so that a formal theory for the continuum case would have a significant practical meaning. Besides that, the features here discussed seem to indicate that a generalized continuum approach is needed for the theory of the homogenized limit of a pantographic sheet, and therefore one cannot underestimate the theoretical significance of the study of a rigorous homogenization procedure for this kind of structures.

Of course, the implicit simplification due to the assumption of ideal hinges has to be replaced, if one wants to proceed towards concrete applications, by more realistic hinges having a nonzero rotational stiffness. Moreover, since the axial stiffness is usually much larger than the bending stiffness, it would be reasonable to consider pantographic sheets made of inextensible fibers as a better approximation of real objects. The theoretical, numerical and experimental investigation of such kind of structures looks indeed a very promising research field at the moment.

\section{Funding statement}

The research was funded by the Italian Ministry of University and Research, under the Scientific Research Program of Relevant National Interest: Year 2010-2011 Protocol 2010MBJK5B-005 End date: 01/02/ 2016, Title 'Dynamics, Stability and Control of Flexible Structures'.

\section{References}

[1] F. dell'Isola, I. Giorgio, U. Andreaus, Elastic pantographic 2D lattices: a numerical analysis on static response and wave propagation, in: Proceedings of the Estonian Academy of Sciences, 64 (3), 2015, 219-225.

[2] A. Madeo, A. Della Corte, L. Greco, P. Neff, Wave propagation in pantographic 2D lattices with internal discontinuities, in: Proceedings of the Estonian Academy of Sciences, 64 (3S), 2015, 325-330.

[3] I. Goda, M Assidi, S, Belouettar, J.F Ganghoffer, A micropolar anisotropic constitutive model of cancellous bone from discrete homogenization, J. Mech. Behav. Biomed. Mater. 16 (2012) 87-108.

[4] F. Dos Reis, J.F. Ganghoffer, Equivalent mechanical properties of auxetic lattices from discrete homogenization, Comput. Mater. Sci. 51 (1) (2012) 314-321. 
[5] F. Dos Reis, J.F. Ganghoffer, Discrete homogenization of architectured materials: implementation of the method in a simulation tool for the systematic prediction of their effective elastic properties, Tech. Mech. 30 (2010) 85-109.

[6] F. Dos Reis, J.-F. Ganghoffer, Construction of micropolar continua from the homogenization of repetitive planar lattices, in: Mechanics of Generalized Continua, Springer, Berlin, 2011, pp. 193-217.

[7] M. Assidi, F. DosReis, J.-F. Ganghoffer, Equivalent mechanical properties of biological membranes from lattice homogenization, J. Mech. Behav. Biomed. Mater. 4 (8) (2011) 1833-1845.

[8] V.A. Eremeyev, E.A. Ivanova, N.F. Morozov, A.N. Solov'ev, On the determination of eigenfrequencies for nanometer-size objects, Dokl. Phys. 51 (2) (2006) 93-97.

[9] V.A. Eremeyev, E.A. Ivanova, N.F. Morozov, A.N. Soloviev, Method of determining the eigenfrequencies of an ordered system of nanoobjects, Tech. Phys. 52 (1) (2007) 1-6.

[10] V.A. Eremeyev, E.A. Ivanova, N.F. Morozov, S.E. Strochkov, The spectrum of natural oscillations of an array of micro-or nanospheres on an elastic substrate, Doklady Phys. 52 (12) (2007) 699-702.

[11] V.A. Eremeyev, E.A. Ivanova, D.A. Indeitsev, Wave processes in nanostructures formed by nanotube arrays or nanosize crystals, J. Appl. Mech. Tech. Phys. 51 (4) (2010) 569-578.

[12] A. Rinaldi, L. Placidi, A microscale second gradient approximation of the damage parameter of quasi-brittle heterogeneous lattices, ZAMM-J. Appl. Math. Mech./Z. Angew., Math. Mech. 94 (10) (2014) 862-877.

[13] Y. Yang, A. Misra, Higher-order stress-strain theory for damage modeling implemented in an element-free Galerkin formulation, CMES-Comput. Model. Eng. Sci. 64 (1) (2010) 1-36.

[14] Y. Yang, W.Y. Ching, A. Misra, Higher-order continuum theory applied to fracture simulation of nanoscale intergranular glassy film, J. Nanomech. Micromech. 1 (2) (2011) 60-71.

[15] C. Pideri, P. Seppecher, A second gradient material resulting from the homogenization of an heterogeneous linear elastic medium, Contin. Mech. Thermodyn. 9 (5) (1997) 241-257.

[16] C. Pideri, P. Seppecher, Asymptotics of a non-planar rod in non-linear elasticity, Asymptot. Anal. 48 (1) (2006) 33-54.

[17] P. Seppecher, J.-J. Alibert, F. dell'Isola, Linear elastic trusses leading to continua with exotic mechanical interactions, J. Phys.: Confer. Ser. 319 (1) (2011) 012018.

[18] F. dell'Isola, P. Seppecher, A. Madeo, How contact interactions may depend on the shape of Cauchy cuts in Nth gradient continua: approach "à la D' Alembert", Z. Angew., Math. Phys. 63 (6), 2012, 1119-1141.

[19] L. Placidi, A variational approach for a nonlinear 1-dimensional second gradient continuum damage model, Contin. Mech. Thermodyn. 27 (4) (2015) 623-638.

[20] A.C. Eringen, Mechanics of Micromorphic Continua, Springer, Freudenstadt and Stuttgart (Germany) 1967, 1968.

[21] J. Altenbach, H. Altenbach, V.A. Eremeyev, On generalized Cosserat-type theories of plates and shells: a short review and bibliography, Arch. Appl. Mech. 80 (1) (2010) 73-92.

[22] S. Federico, A. Grillo, W. Herzog, G. Giaquinta, S. Imatani, Possible approaches in modelling rearrangement in a microstructured material, Key Eng. Mater. 340 (2007) 137-142.

[23] N. Hamila, P. Boisse, Tension locking in finite-element analyses of textile composite reinforcement deformation, C. R. Méc. 341 (6) (2013) 508-519.

[24] N. Hamila, P. Boisse, Locking in simulation of composite reinforcement deformations. analysis and treatment, Compos. Part A: Appl. Sci. Manuf. 53 (2013) 109-117.

[25] A. Cazzani, M. Malagù, E. Turco, Isogeometric analysis: a powerful numerical tool for the elastic analysis of historical masonry arches, Contin. Mech. Thermodyn. http://dx.doi.org/10.1007/s00161-014-0409-y.

[26] A. Cazzani, M. Malagù, E. Turco, Isogeometric analysis of plane-curved beams, Math. Mech. Solids, http://dx.doi.org/10.1177/1081286514531265.
[27] L. Greco, M. Cuomo, B-Spline interpolation of Kirchhoff-Love space rods, Comput. Methods Appl. Mech. Eng. 256 (2013) 251-269.

[28] L. Greco, M. Cuomo, An implicit G1 multi patch B-spline interpolation for Kirchhoff-Love space rod, Comput. Methods Appl. Mech. Eng. 269 (2014) 173-197.

[29] A. Cazzani, P. Ruge, Numerical aspects of coupling strongly frequencydependent soil-foundation models with structural finite elements in the time-domain, Soil Dyn. Earthq. Eng. 37 (2012) 56-72.

[30] E. Turco, P. Caracciolo, Elasto-plastic analysis of Kirchhoff plates by high simplicity finite elements, Comput. Methods Appl. Mech. Eng. 190 (5) (2000) 691-706.

[31] V.A. Yeremeyev, A.B. Freidin, L.L. Sharipova, Non-uniqueness and stability in problems of the equilibrium of elastic two-phase solids, Dokl. Ross Akad. Nauk. 391 (2) (2003) 189-193.

[32] A. Luongo, D. Zulli, G. Piccardo, A linear curved-beam model for the analysis of galloping in suspended cables, J. Mech. Mater. Struct. 2 (4) (2007) 675-694.

[33] A. Luongo, A. Paolone, G. Piccardo, Postcritical behavior of cables undergoing two simultaneous galloping modes, Meccanica 33 (3) (1998) 229-242.

[34] G. Piccardo, L.C. Pagnini, F. Tubino, Some research perspectives in galloping phenomena: critical conditions and post-critical behavior, Contin. Mech. Thermodyn. 27 (1-2) (2015) 261-285.

[35] A. Luongo, G. Piccardo, Linear instability mechanisms for coupled translational galloping, J. Sound Vib. 288 (4) (2005) 1027-1047.

[36] A. Cazzani, On the dynamics of a beam partially supported by an elastic foundation: an exact solution-set, Int. J. Struct. Stab. Dyn. 13 (08) (2013) 1350045, http://dx.doi.org/10.1142/S0219455413500454 [30 pages].

[37] M. Bîrsan, H. Altenbach, T. Sadowski, V.A. Eremeyev, D. Pietras, Deformation analysis of functionally graded beams by the direct approach, Compos. Part B: Eng. 43 (3) (2012) 1315-1328.

[38] U. Andreaus, R.C. Batra, M. Porfiri, Vibrations of cracked Euler-Bernoulli beams using meshless local Petrov-Galerkin (mlpg) method, CMES-Comput. Model. Eng. Sci. 9 (2) (2005) 111-131.

[39] U. Andreaus, P. Casini, F. Vestroni, Nonlinear features in the dynamic response of a cracked beam under harmonic forcing, in: ASME 2005 International Design Engineering Technical Conferences and Computers and Information in Engineering Conference, American Society of Mechanical Engineers, Long Beach, California, USA, 2005, pp. 2083-2089.

[40] J. Cao, R. Akkerman, P. Boisse, J. Chen, H.S. Cheng, E.F. De Graaf, J.L. Gorczyca, P. Harrison, G. Hivet, J. Launay, W. Lee, L. Liu, S.V. Lomov, A. Long, E. de Luycker, F. Morestin, J. Padvoiskis, X.Q. Peng, J. Sherwood, T. Stoilova, X.M. Tao, I. Verpoest, A. Willems, J. Wiggers, T.X. Yu, B. Zhu, Characterization of mechanical behavior of woven fabrics: experimental methods and benchmark results, Compos. Part A: Appl. Sci. Manuf. 39 (6) (2008) 1037-1053.

[41] H. Nikopour, A. Selvadurai, Concentrated loading of a fibre-reinforced composite plate: experimental and computational modeling of boundary fixity, Compos. Part B: Eng. 60 (2014) 297-305, http://dx.doi.org/10.1016/j. compositesb.2013.12.034.

[42] A. Salupere, J. Engelbrecht, Hidden and driven solitons in microstructured media, in: 21st International Congress of Theoretical and Applied Mechanics ICTAM04 CD-ROM Proceedings. IPPT PAN, Warszawa, 2004, pp. 1-2.

[43] A. Madeo, P. Neff, I.-D. Ghiba, L. Placidi, G. Rosi, Band gaps in the relaxed linear micromorphic continuum, ZAMM-J. Appl. Math. Mech./Z. Angew. Math Mech., 95 (9), 2015, 880-887. http://dx.doi.org/10.1002/zamm.201400036.

[44] A. Sestieri, A. Carcaterra, Space average and wave interference in vibration conductivity, J. Sound Vib. 263 (3) (2003) 475-491.

[45] A. Carcaterra, N. Roveri, G. Pepe, Fractional dissipation generated by hidden wave-fields, Math. Mech. Solids 20 (10), 2015, 1251-1262. http://dx.doi.org/10. $1177 / 1081286513518941$. 